\title{
Family well-being is valued more than personal well-being: A four-country study
}

\author{
Kuba Krys ${ }^{1,2}$ (D) Colin A. Capaldi ${ }^{3}$ John M. Zelenski ${ }^{3} \cdot$ Joonha Park $^{4} \cdot$ Martin Nader $^{5} \cdot$ Agata Kocimska-Zych $^{6}$. \\ Anna Kwiatkowska ${ }^{1} \cdot$ Piotr Michalski $^{1} \cdot$ Yukiko Uchida $^{2}$
}

Published online: 27 April 2019

(C) The Author(s) 2019

\begin{abstract}
Well-being is recognized as a fundamental human goal and a universal human aspiration. However, some cross-country studies suggest that the desirability of the most often studied concept of well-being - personal life satisfaction — varies across countries, and we know little about the desirability of other types of well-being. Extending this novel area of research, we argue that focusing on the family (as compared to the individual) as the subject of well-being may be another important distinction in how well-being is conceptualized and valued. With data collected in four countries that tend to occupy different positions in rankings of personal life satisfaction (i.e., Canada, Colombia, Japan, and Poland), we document that, irrespective of cultural context, family well-being is valued over personal well-being. These findings suggest that policy makers and scientists may need to pay more attention to family well-being than they currently do.
\end{abstract}

Keywords Family $\cdot$ Happiness $\cdot$ Well-being $\cdot$ Interdependent happiness $\cdot$ Life satisfaction $\cdot$ Cultural psychology

The recognition of well-being as an ultimate aim of societal development is becoming increasingly widespread. In 2011, the UN recognized well-being as a fundamental human goal and a universal human aspiration. The implementation of national accounts of well-being seems to be one of the options gaining traction in the search for new indicators of societal functioning (Adler and Seligman 2016; Diener et al. 2015; Helliwell et al. 2016). We propose that if the complexity of human flourishing is to be represented in these novel indices,

Electronic supplementary material The online version of this article (https://doi.org/10.1007/s12144-019-00249-2) contains supplementary material, which is available to authorized users.

Kuba Krys

kuba@krys.pl

1 Institute of Psychology, Polish Academy of Sciences, Jaracza 1, 00-378 Warsaw, Poland

2 Kokoro Research Center, Kyoto University, Kyoto, Japan

3 Department of Psychology, Carleton University, Ottawa, Canada

4 Nagoya University of Commerce and Business, Nagoya, Japan

5 Department of Psychological Studies, Universidad ICESI, Cali, Colombia

6 Department of Psychological Studies, SWPS University, Wrocław, Poland researchers may need to understand the diverse ways in which people conceptualize and desire well-being. Whose well-being is valued more: the well-being of individuals or the well-being of families (Krys et al. 2019; Newland 2015)? Which type of well-being is valued more: more achievement-oriented well-being (e.g., life satisfaction; Diener et al. 1985) or more relationship-oriented well-being (e.g., interdependent happiness; Hitokoto and Uchida 2015)? From a cross-cultural perspective, answers to these questions seem to be lacking, and the main purpose of the current paper is to address them.

While previous cross-country research has focused mainly on personal life satisfaction, we posit that across countries people may value other aspects of well-being as well. In particular, family well-being is a type of well-being that we predict will be highly valued. Here, we offer a preliminary examination and comparison of the desirability of family and personal well-being, and we investigate how this might relate to the experience of well-being. Additionally, we differentiate between two types of well-being: the traditionally researched concept of life satisfaction (Diener et al. 1985) and the more Eastern concept of interdependent happiness (Hitokoto and Uchida 2015). As a result, we compare the valuation and levels of four different types of well-being, ranging from a more traditional individualism-themed conceptualization of well-being (i.e., life satisfaction of an individual) to a more collectivism-themed one (i.e., interdependent happiness of a 
family; see Fig. 1 for their classification). We carried out our study in four different countries occupying quite different positions in Diener et al.'s (1995) ranking of levels of personal life satisfaction: Canada and Colombia (high positions), and Japan and Poland (low positions). This way we hope to examine what is shared among people from various cultures, and what is specific to certain cultural contexts.

We study family well-being from an intra-personal perspective (Krys et al. 2019); that is, we study how a single individual judges the well-being of his/her family as a whole (which can be complementary to an interpersonal perspective; e.g., judgments of family well-being averaged or agreed between members of a family). Similar to Diener et al. (1985), we define family life satisfaction as a person's global assessment of their family's quality of life according to this person's own criteria. Similar to Hitokoto and Uchida (2015), we define family interdependent happiness as a global subjective assessment of whether a person's family is socially harmonized with other people, quiescent, ordinary, and connected to the collective way of well-being. In instances when the differentiation between life satisfaction and interdependent happiness is not necessary and we wish to refer to both, we use the umbrella term 'well-being'.

\section{Well-Being Valuation Varies across Cultures}

Some view personal life satisfaction as the ultimate value in life (Lu and Gilmour 2004). A belief that people should explicitly and actively pursue their own life satisfaction is strongly endorsed in the United States of America (Baker 2014; Lu and Gilmour 2004), and the pursuit of happiness more generally is enshrined in the Declaration of
Independence as one of three inalienable rights. Such positive evaluations of life satisfaction are common among individuals shaped by Western, Educated, Industrialized, Rich and Democratic societies (WEIRD; Henrich et al. 2010), and are implicitly assumed to apply to the whole human race. In contrast, some psychological research hints that well-being may be defined, experienced, and valued differently across individuals and cultures (Delle Fave et al. 2016; Hornsey et al. 2018; Lu and Gilmour 2004; Uchida and Oishi 2016). For example, in contrast to researchers' typical focus on life satisfaction, a more open-ended approach by Delle Fave and collaborators (2016) revealed that harmony and balance were mentioned more often than satisfaction among lay definitions of happiness. Similarly, analysis of extracts from essays written on 'what is happiness?' reveals that the prioritization of life satisfaction is not as popular among East Asians as among members of WEIRD societies (Lu and Gilmour 2004).

Analysis of countries that voted on UN resolution 65/309, titled 'Happiness: Towards a Holistic Approach to Development', delivers further evidence that well-being may not be universally attributed the highest importance across all cultures. Although the UN adopted the resolution without opposing votes, only 66 out of $193 \mathrm{UN}$ members supported it (Koh 2014). Koh found that wealthier and individualistic societies made up a disproportionate number of countries who voted in favor. More recently, Hornsey and collaborators (2018) documented that self-reported ideal levels of happiness are lower among members of holistic cultures than members of other cultures. Similarly, Diener et al. (2000) found that personal life satisfaction was valued across the forty-one countries they studied, but there was significant cultural variation in the degree to which personal life
Fig. 1 Four kinds of well-being and their measures. The vertical axis differentiates the subject of measure (an individual vs. a family), and the horizontal axis differentiates the type of well-being (life satisfaction vs. interdependent happiness)

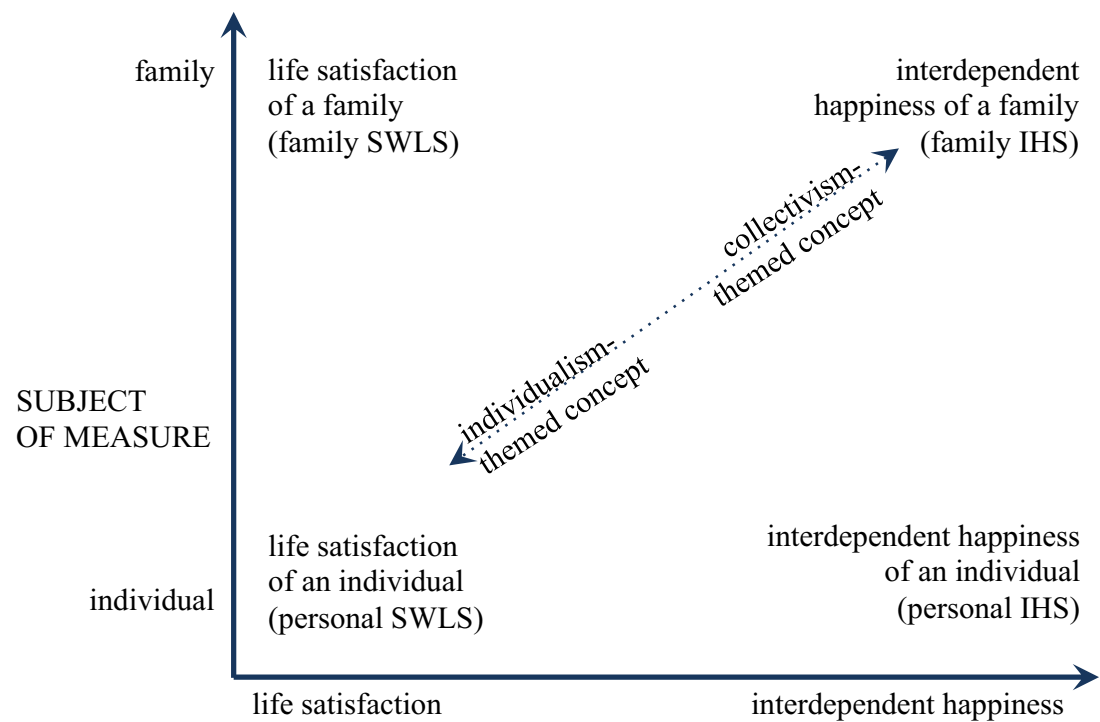

TYPE OF WELL-BEING 
satisfaction was valued. Whereas Latin American and individualistic cultures almost hit the ceiling of the personal life satisfaction valuation scale, Japan, Korea, and China were around the neutral midpoint of the scale.

Results from Diener et al. (2000) suggest that societies differ in how much they value the most often studied aspect of well-being (i.e., personal life satisfaction). Given this, researchers might want to attempt to identify a subjective wellbeing measure that will be more cross-culturally generalizable (i.e., valued highly across all cultures). However, such a single 'universal' measure may be difficult to find. We find it more plausible that variation in cultural values will translate into divergent views of the good life. Societies may wish to flourish in their own indigenously defined way (see for example the Bhutanese Gross National Happiness Index; Ura et al. 2012). Thus, the role of scientists may be to study which kind of well-being is valued in a given culture and why. Here, we bridge this gap by comparing the valuation of individual- and family-oriented well-being in their Western and Eastern forms (i.e., life satisfaction and interdependent happiness). We assume that valuations of specific kinds of well-being can be important antecedents of people's motivation towards achieving them.

\section{Family Well-Being}

The majority of studies in psychology assess, and focus on, individuals (Bond 2002). Cross-cultural studies, however, document that the individual person is the basic reference point in only some cultures (labelled individualistic), whereas being a part of a group dominates the thinking and behavior of people living in the majority of societies (labelled collectivistic; Hofstede 2001). The dominant psychological approach that isolates and disembeds ' $\mathrm{I}$ ' from the relational context may be regarded as an individualistic cultural legacy in academia (Sampson 1981). In collectivistic cultures, others are relatively more focal in one's own behavior. Thus, selfdescriptions in psychology may need to be extended with $u s$ descriptions - that is, descriptions of the basic social groups people identify with (e.g., Krys et al. 2019).

Among the many groups that people belong to, the family probably has the greatest personal importance for many individuals (Cousins 1989; Lee et al. 2012) _-indeed, family is arguably the fundamental and the oldest unit of societies (DeFrain and Asay 2007; Krys et al. 2019). Data collected in the World Values Survey (WVS; 2016) confirm that among six aspects of life-family, friends, leisure time, politics, work, and religion-family is rated as the most important in the over sixty analyzed countries; in no single country is another aspect of life rated as more important than family (see Fig. 2 for illustration). The WVS data, however, do not allow one to explicitly compare the valuation of family well-being to personal well-being as we do in the current study.

In family well-being research, concepts, constructs, and measures often originate in personal well-being studies ( $\mathrm{Hu}$ et al. 2011; Krys et al. 2019; Park et al. 2003; Zabriskie and McCormick 2003; Zabriskie and Ward 2013). Here, we adopt a similar approach and conceptualize family life satisfaction and family interdependent happiness as family-level constructs emerging from personal well-being constructs: life satisfaction (Diener et al. 1985) and personal interdependent happiness (Hitokoto and Uchida 2015; see also Fig. 1). In the literature, the meaning of family well-being varies: it is conceptualized either in a narrow way covering specific areas relevant to family well-being (e.g., family self-sufficiency, family resiliency, marital issues, or work-life interferences; Andersz et al. 2018; Chien and Mistry 2013; Newland 2015), or globally as generalized family well-being (e.g., "conscious cognitive judgment of one's family life in which the criteria for the judgment are up to the individual" [Zabriskie and Ward 2013, p. 449]). In our study, we adhere to a general conceptualization of family well-being (i.e., its globalized and holistic assessment). The most often studied perspective is the one of a single family member ${ }^{1}$ (e.g., Family Satisfaction Scale, Olson and Wilson 1982; Family APGAR, Smilkstein 1978; see review by $\mathrm{Hu}$ et al. 2011), and we adopt the perspective of a single member of a family too.

However, unlike most research on family well-being, in our conceptualization we make family the central subject of wellbeing. Instead of researching how individuals are satisfied with their family life, we study how families are satisfied with their life (Krys et al. 2019). This novelty let us directly study the valuation of well-being of family as a whole (cf. the valuation of being satisfied with one's own family). The well-being of a family as a whole is fundamentally important to thriving individuals and societies (McKeown and Sweeney 2001), for positive parenting and child well-being (Newland 2015), and for couples (Johnson et al. 2006; Kamp Dush et al. 2008).

\section{Life Satisfaction and Interdependent Happiness}

Depending on the context and the culture, people define wellbeing in various ways (e.g., life satisfaction, interdependent happiness, positive emotions, lack of negative emotions, contentment, harmony, self-actualization, self-autonomy, strong relationships, relationship flourishing, optimism, achievement, health, leisure, hedonism, eudaimonia; Delle Fave

\footnotetext{
${ }^{1}$ The perspective of more than one person is rare and rather limited to research of families with individuals whose perspective can be difficult to study (e.g., families with small children or with disabled family members; Bowman 2001; Brown et al. 2006).
} 


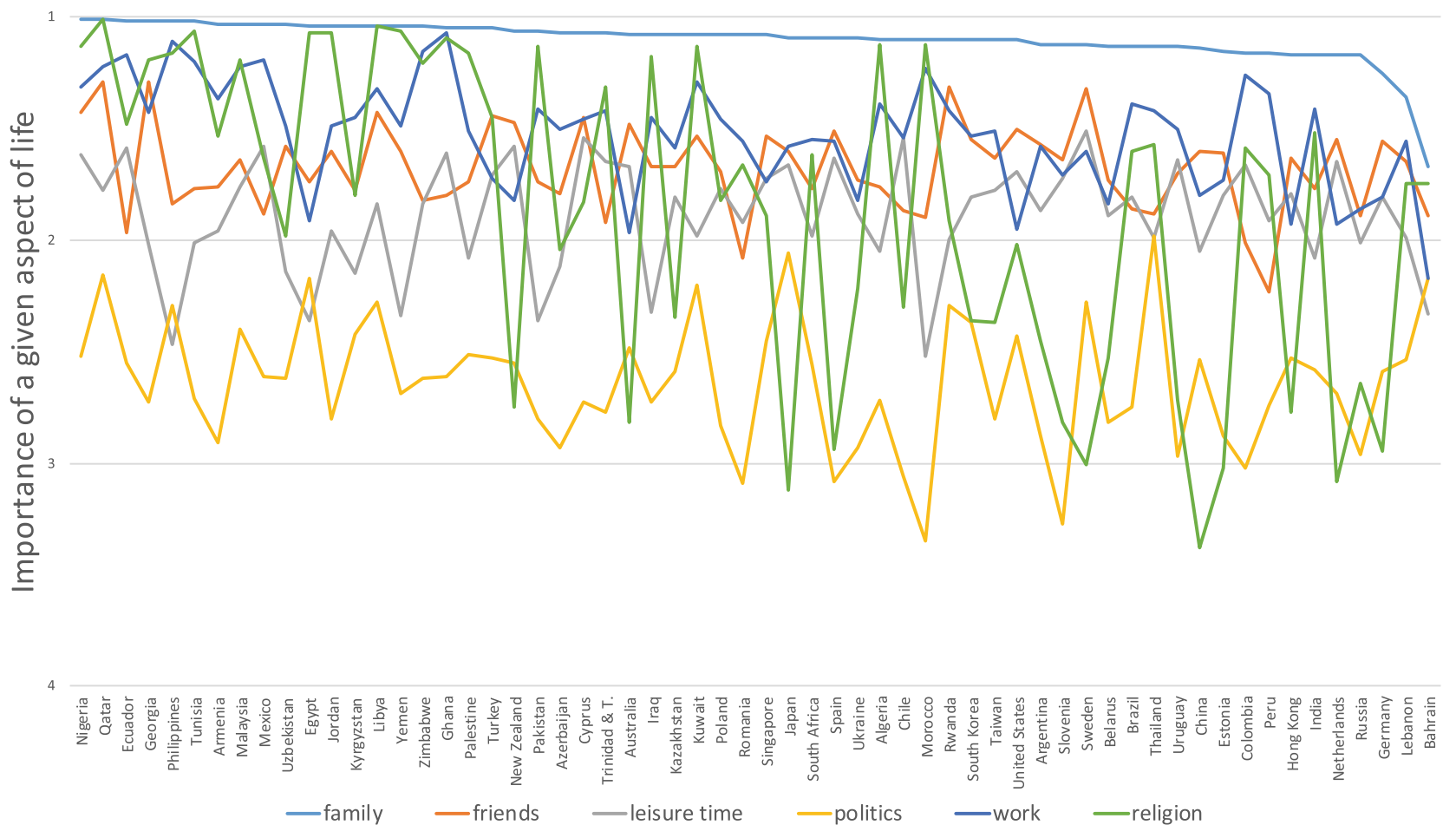

Fig. 2 Importance of six aspects of life across sixty countries analyzed in the World Values Survey (2016). The scale ranges from 1 (very

important) to 4 (not at all important). Family was attributed the highest importance in all sixty analyzed countries

et al. 2016; Fowers et al. 2016; Uchida and Oishi 2016). In most of the studies on well-being as a measure of societal development, an arguably Euro-American personally-oriented definition of well-being-focusing on personal life satisfaction-has been adopted (Adler and Seligman 2016; Diener et al. 2015; Helliwell et al. 2016; Krys et al. 2018). Thus, in order to broaden the understanding of the cultural variation of well-being desirability, we decided to contrast the dominating individualistic understanding of well-being with a more socially-oriented well-being that comes from an East Asian perspective (Hitokoto and Uchida 2015; Krys et al. 2019; Lu and Gilmour 2004).

Japanese researchers Hitokoto and Uchida (2015) introduced the concept of interdependent happiness, which they defined as 'the global, subjective assessment of whether one is interpersonally harmonized with other people, being quiescent, and being ordinary, and connected to the collective way of well-being' (p. 214). Interdependent happiness contrasts with traditional measures of well-being that have dominated research in positive psychology. In particular, Diener and collaborators' (1985) Satisfaction With Life Scale (SWLS) has become the model scale for many studies, including large crosscultural studies carried out on representative national samples (e.g., the WVS). Many Western conceptualizations of wellbeing are based on aspects of individual functioning like autonomy, environmental mastery, personal growth, purpose in life, and self-acceptance (Ryff and Keyes 1995; Uchida and Kitayama 2009). Although strong personal relationships are also included in Western conceptualizations, they contrast with the more interdependent happiness rooted in Eastern traditions where interpersonal relationships are central, and a state of harmony and balance between the self and significant others are most important (Hitokoto and Uchida 2015).

Delle Fave and collaborators' (2016) study on lay understanding of well-being across twelve countries documents that East Asian (i.e., socially-oriented) conceptualizations of wellbeing may be more universal than many traditional approaches taken by Western psychologists (i.e., individuallyoriented). As Delle Fave and colleagues write:

Over and above differences related to country membership, cultural dimensions, and demographic features, the findings $[\ldots]$ highlight a substantial similarity across countries in the core definitions of happiness. At the psychological level, happiness was predominantly identified as inner harmony, a balanced and positive connectedness perceived among various facets of the self. [...] At the contextual level, positive and harmonious family and social relationships were described across countries as key components of happiness. (p. 19) 
Thus, views of well-being based on social harmony and interdependence (emphasized by East Asian researchers) seem to be shared around the globe, whereas well-being derived from individual functioning seems to be a more Euro-American perspective. Family, in particular, plays an important role among contextual definitions of well-being across all analyzed cultures.

\section{Present Study}

The main purpose of the current study is to answer the question of whose well-being people value more: their own personal well-being or their family's well-being? As the body of studies on well-being grows, we found this question fundamental. Although well-being seems to be desired by the majority of people, studies on valuation of personal life satisfaction suggest that the valuation of this specific type of well-being is not equally high across countries (Diener et al. 2000; Hornsey et al. 2018). Individualistic countries seem to value personal life satisfaction more than collectivistic countries (Koh 2014). In the current study, we tested how people in four different countries value four different types of well-being. While there are surely many factors that contribute to the cultural variety in well-being valuation, we selected two variables that seem important in a cross-cultural comparison of well-being desirability: (1) the subject of a measure (individuals vs. their families; Krys et al. 2019), and (2) the type of well-being (interdependent happiness [a concept introduced to psychology by East Asian researchers] vs. life satisfaction [a concept introduced to psychology by researchers from WEIRD societies]). In Fig. 1, we present the classification of the four emergent concepts of well-being: (1) life satisfaction of the individual, (2) life satisfaction of the family, (3) interdependent happiness of the individual, and (4) interdependent happiness of the family. Out of the four types of wellbeing we examine, life satisfaction of the individual is the most individualism-themed, and interdependent happiness of the family is the most collectivism-themed; life satisfaction of the family and interdependent happiness of the individual can be regarded as mixing different qualities of both contexts. That is, family life satisfaction focuses on a subject that is more collectivistic (i.e., one's family) but a type of well-being that is more individualistic (i.e., life satisfaction), while individual interdependent happiness focuses on a subject that is more individualistic (i.e., the individual) but a type of well-being that is more collectivistic (i.e., interdependent happiness).

Theorizing and studies on valuation of well-being are novel (for exceptions see Diener et al. 2000; Hornsey et al. 2018; Koh 2014). Thus, we decided to avoid formulating precise theory-driven predictions and took a more exploratory approach. Recognizing the growing literature on the significance of well-being (Adler and Seligman 2016; Diener et al. 2015; Helliwell et al. 2016; also see the UN resolution 65/309), we found it important to answer the question 'Whose well-being do people value more?'.2

\section{Method}

\section{Participants and Selection of Cultures}

To provide a cross-cultural analysis of the valuation of four kinds of well-being (i.e., life satisfaction vs. interdependent happiness $\times$ well-being of an individual vs. well-being of a family), we collected and analyzed data from nonprobabilistic convenience samples in four countries: Canada, Colombia, Japan, and Poland. Post-secondary students from various fields of study were recruited at each team's university. As a rule of thumb, we aimed to collect data from 200 participants from each culture, although some authors managed to collect more and one sample is smaller. Power analyses revealed that our study was adequately powered (see online supplementary material). After team leaders excluded obviously problematic data (e.g., from participants who dropped out after giving their consent or had a lot of missing variables), there were 842 respondents in total. The mean age of respondents was 20.13 years $(S D=3.43)$. Across the four samples, there were 446 female participants (53.0\%), 389 male participants $(46.2 \%)$, one participant who indicated 'other' gender $(0.1 \%)$, and six participants who did not reveal their gender $(0.7 \%)$. Statistics for each country are presented in Table 1. Data were collected in 2016 and 2017. The study received ethical approval at each university.

We aimed to conduct the study in countries that occupy different positions in rankings of personal well-being. We decided to collect data in Canada and Colombia as they tend to have high levels of personal life satisfaction (over $1 S D$ above $M$; Diener et al. 1995), and are examples of individualistic and collectivistic countries respectively (Hofstede 2001). Furthermore, we collected data in individualistic Poland and collectivistic Japan (Hofstede 2001) as both of these countries tend to rank relatively low on personal life satisfaction (more than $.60 S D$ below $M$; Diener et al. 1995).

\section{Materials and Procedure}

In order to measure valuation of the four kinds of well-being, we followed the approach of Diener and collaborators (2000), who asked participants to rate their ideal level of personal life

\footnotetext{
${ }^{2}$ Additionally, we investigated how valuations of specific kinds of well-being predict actual levels of respective kinds of well-being (e.g., to what extent does valuing personal interdependent happiness predict actual levels of personal interdependent happiness), and explored the relation between the valuation of the analyzed concepts of well-being and markers of individualism (i.e., self-construals; Markus and Kitayama 1991; Singelis 1994). We report these results in the online supplementary material.
} 


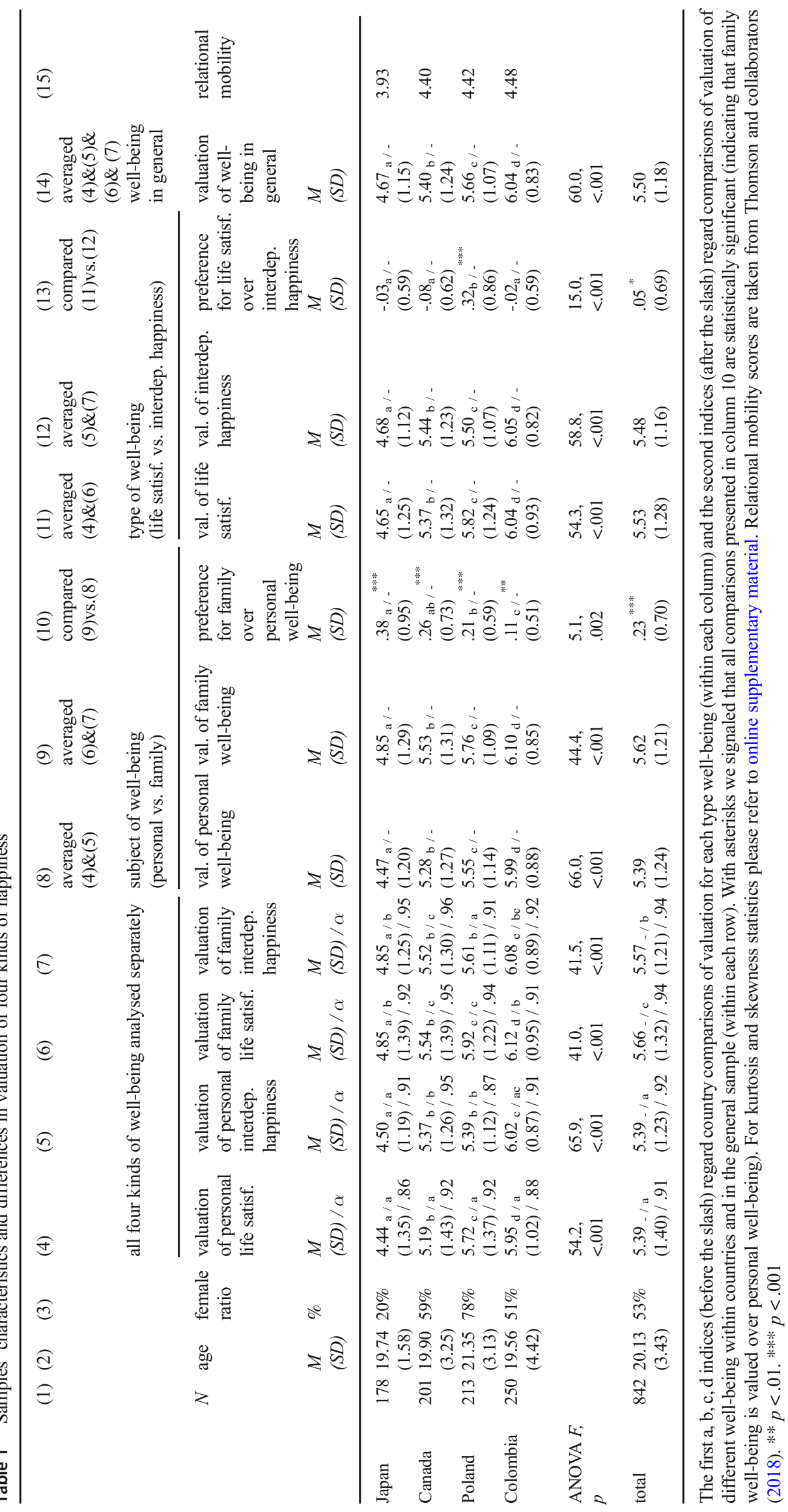


satisfaction. Our instruction, taken from Diener and collaborators, read as follows: [...] instead of answering how much you agree with the statements, we would like you to indicate how much you think the ideal or perfect person would agree with each statement. We used items from the Satisfaction with Life Scale (personal SWLS; Diener et al. 1985; e.g., In most ways my life is close to my ideal) to measure the valuation of life satisfaction of individuals. The items from the Interdependent Happiness Scale (personal IHS; Hitokoto and Uchida 2015; e.g., I can do what I want without causing problems for other people) were used to measure the valuation of interdependent happiness of individuals. We also adapted both above measures to assess participants' views of their families by changing the subject of the personal SWLS and personal IHS measures from an individual to their family (family SWLS and family IHS; e.g., In most ways the life of my family is close to ideal for family SWLS and As a family we can do what we want without causing problems for other people for family IHS; for a full list of the original and modified items see online supplementary material). Zabriskie and McCormick (2003) adopted a similar approach in their Satisfaction With Family Life scale by replacing the word 'life' with 'family life' in each of the original SWLS items.

For each item, participants indicated how much an ideal person would agree using a Likert scale that ranged from 1 (Ideal person wouldn't agree at all) to 7 (Ideal person would fully agree). All scales of well-being valuation were reliable with $\alpha$ s ranging from .86 to .96 (see Table 1 for details and see Table S1 in online supplementary material for equivalence analysis). We also administered scales measuring actual levels of the four types of well-being (actual personal SWLS, actual personal IHS, actual family SWLS, actual family IHS), ${ }^{3}$ and the original 30-item Self-Construal Scale (SCS) proposed by Singelis (1994) that measures independent and interdependent self-construals. We briefly report findings based on these scales in the online supplementary material (but because the collected self-construal data were far from equivalence, conclusions based on these results remain to be confirmed in further studies).

At the end of the questionnaire, all participants were asked to provide information on their sociodemographic background (see Table 1, as well as Table S7 in the online supplementary material). Materials were prepared in English. Instructions and demographic items were translated from English into the language of every country covered by the study, and the appropriate language versions of the SWLS and IHS (and SCS) scales were used. In order to establish linguistic equivalence, team leaders were asked to follow the back-translation procedure for the instructions and sociodemographic questionnaire

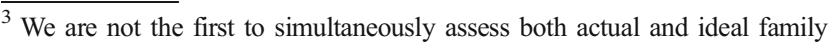
well-being (see Diener et al. 2000; Olson et al. 1985).
}

(Brislin 1970). The presentation order of the items and scales were kept consistent across individuals and countries.

\section{Results}

A mixed-design ANOVA was conducted with type of valued well-being (life satisfaction vs. interdependent happiness) and subject of valued well-being (individual vs. family) as withinsubject factors, and country (Japan vs. Canada vs. Poland vs. Colombia) and gender (female vs. male) as between-subjects factors. We included gender to make sure that presented effects are not biased by gender imbalance in our samples. Participants with missing data on gender and the participant who indicated 'other' were excluded from this ANOVA. Overall results from this ANOVA (i.e., testing main effects and interactions) are presented in Table 2, while Table 1 presents results for each country and includes more detailed follow-up comparisons of happiness valuation within and between countries. The main effect of country $\left(\eta_{\mathrm{p}}{ }^{2}=.14\right)$ indicated that valuation of well-being significantly varies across countries (see column 14 in Table 1). Like in Diener and colleagues' (2000) study on valuation of personal life satisfaction, Japanese participants in our study indicated that they valued well-being somewhat above the middle-point of the scale $\left(M_{\mathrm{Japan}}=4.67, S D_{\mathrm{Japan}}=1.15\right)$, whereas Colombian participants valued well-being close to the ceiling of the scale $\left(M_{\text {Colombia }}=6.04, S D_{\text {Colombia }}=0.83\right)$. Valuations of wellbeing significantly differed between all four analyzed countries, and the difference was very large in the case of the countries valuing well-being the least and the most (Japan and Colombia), $t(426)=14.46, p<.001, d=1.19$.

The main effect of subject of well-being $\left(\eta_{\mathrm{p}}^{2}=.09\right)$ showed that participants rated family well-being significantly higher than personal well-being $\left(M_{\text {Family }}=5.62, S D_{\text {Family }}=1.21\right.$; $M_{\text {Personal }}=5.39, S D_{\text {Personal }}=1.24 ; t[834]=9.38, p<.001, d_{r m-}$ $=.26$; see columns $8-10$ in the bottom row of Table 1). To illustrate this effect in a simple way, $26 \%$ of participants valued personal well-being over family well-being, $16 \%$ of participants valued both well-beings equally, and the majority of participants (58\%) valued family well-being over personal well-being (Fig. 3 presents these statistics split up by country). Finally, the main effect of type of well-being $\left(\eta_{\mathrm{p}}{ }^{2}<.01\right)$ indicated that life satisfaction was valued significantly (albeit slightly) more than interdependent happiness $\left(M_{\text {LifeSatisfaction }}=5.53, S D_{\text {LifeSatisfaction }}=1.28\right.$; $M_{\text {InterdependentHappiness }}=5.48, S D_{\text {InterdependentHappiness }}=1.16$; $t[837]=2.09, p=.037, d_{r m}=.06$; see columns $11-13$ in the bottom row of Table 1). The main effect of gender was not statistically significant.

\footnotetext{
${ }^{4}$ When calculating Cohen's $d$ for repeated measures effects, we used equation 9 from Lakens (2013).
} 
Table 2 Results of ANOVA for valuation of various forms of well-being

\begin{tabular}{lllll}
\hline & $F$ & $d f$ & $p$ & $\eta_{p}{ }^{2}$ \\
\hline $\begin{array}{l}\text { type of well-being (TYPE) } \\
\text { (life satisfaction vs. interdependent happiness) }\end{array}$ & 4.91 & 1,827 & .027 & .006 \\
subject of well-being (SUBJECT) & & & & \\
(personal well-being vs. family well-being) & 80.95 & 1,827 & $<.001$ & .089 \\
country (C) & & & & \\
gender (G) & 44.18 & 3,827 & $<.001$ & .138 \\
INTERACTIONS: & 0.05 & 1,827 & .83 & .000 \\
TYPE x SUBJECT & & & & \\
TYPE x C & 12.66 & 1,827 & $<.001$ & .015 \\
TYPE x G & 10.47 & 3,827 & $<.001$ & .037 \\
C x G & 1.11 & 1,827 & .29 & .001 \\
TYPE x C x G & 0.88 & 3,827 & .45 & .003 \\
SUBJECT x C & 0.99 & 3,827 & .40 & .004 \\
SUBJECT x G & 3.80 & 3,827 & .010 & .014 \\
SUBJECT x C x G & 1.25 & 1,827 & .26 & .002 \\
TYPE x SUBJECT x C & 0.50 & 3,827 & .69 & .002 \\
TYPE x SUBJECT x G & 1.62 & 3,827 & .18 & .006 \\
TYPE x SUBJECT x C x G & 3.82 & 1,827 & .051 & .005 \\
\hline
\end{tabular}

More importantly, we observed three significant two-way interactions. The largest interaction effect $\left(\eta_{\mathrm{p}}{ }^{2}=.04\right)$ we found was for the type of valued well-being by country. Thus, we compared the valuation of life satisfaction and interdependent happiness for each country separately (see columns 11-13 in Table 1), and found that there was a statistically significant difference in Poland between the valuation of the two types of well-being (Poland: $M_{\text {LifeSatisfaction }}=5.82, S D_{\text {LifeSatisfaction }}=$ $1.24 ; M_{\text {InterdependentHappiness }}=5.50, S D_{\text {InterdependentHappiness }}=$ $1.07 ; t[212]=5.36, p<.001, d_{r m}=.31$ ), whereas no significant differences were found between valuations of type of well-being in the other analyzed countries (for Canada $p=.079$; two other $p \mathrm{~s}>.50$ ). This suggests that only one country (i.e., Poland) was responsible for the main effect of type of well-being, and that interdependent happiness tends to not be valued less than the most often studied life satisfaction in the three other analysed countries.

To understand the second observed two-way interaction-for subject of well-being by country $\left(\eta_{\mathrm{p}}{ }^{2}=.01\right)$ - we compared the valuation of family and personal well-being in each country separately. We found that family well-being was valued more than personal wellbeing in each analyzed sample, although the size of this gap varied slightly between countries: from $d_{r m}=.25$ in Colombia to $d_{r m}=.32$ in Japan and Poland (for details see columns 8-10 in Table 1). Thus, preference for family well-being was observed in all four analyzed countries, but with somewhat different intensity.

Fig. 3 Valuation of personal and family well-being across our samples. The red (i.e., left) bars show the percentage of participants valuing personal over family well-being, the blue (i.e., middle) bars show the percentage of those equally valuing both, and the orange (i.e., right) bars show the percentage of participants valuing family over personal well-being

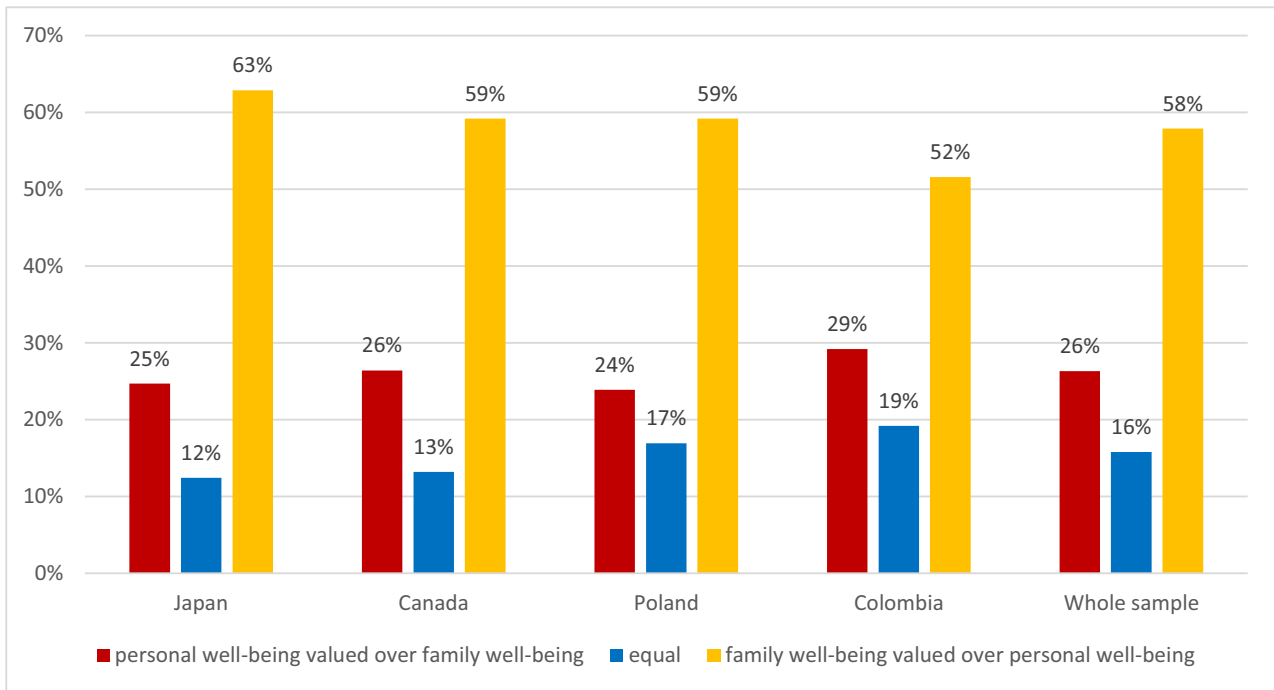


To illustrate the third significant two-way interaction, which we found for type of well-being by subject of wellbeing $\left(\eta_{\mathrm{p}}{ }^{2}=.02\right)$, we compared valuations of all four kinds of well-being with each other. We found that valuation of both types of personal well-being (personal life satisfaction and personal interdependent happiness) did not significantly differ overall, $t(837)=.22, p=.83$, whereas comparisons of other valuations of well-beings were significantly different, $t$ $\mathrm{s}(837)>3.77, p \mathrm{~s}<.001$. This means that when it came to personal well-being, both life satisfaction and interdependent happiness were similarly valued. The most valued kind of well-being was family life satisfaction, followed by family interdependent happiness, with personal life satisfaction and personal interdependent happiness being valued the least (see columns 4-7 in the bottom row of Table 1).

\section{Discussion}

The most important novelty of our study is that we show that the subject of well-being is an important factor in understanding well-being valuation. In all four analyzed countries, family well-being is valued more than personal well-being. Although the effect sizes were rather small, they were relatively consistent across samples - around two times more participants valued family over individual well-being than individual over family well-being (see Fig. 3). Whereas psychological research habitually treats personal well-being as the 'ultimate' dependent variable, we document that family well-being may be more important for people. Future studies may need to recognize and account for this. Our finding is consistent with previous studies documenting that family is rated as being very important by people around the world (see Fig. 2) and that family plays an important role in conceptualizations of happiness (Delle Fave et al. 2016; Krys et al. 2019). Importantly, our finding also shows that people across various cultures acknowledge that the well-being of us (i.e., one's family) can be more important than the well-being of $I$ (i.e., oneself).

We also show that the gap between family and personal well-being valuation varies somewhat across countries. Differences in relational mobility may be a (post hoc) explanation for this finding (Thomson et al. 2018). In low relationally mobile societies, relationships are mostly fixed-people engage in stable and long-lasting relationships, but their choice of relational partners is relatively limited. In high relationally mobile societies, relationship options are 'richer'people more easily seek out new partners and leave old friends behind. In our study, we found the smallest gap between valuation of family and personal well-being in Colombia-the country in our study with the highest relational mobility. In contrast, the largest gap we found was in Japan, which is a society with relatively low relational mobility (see column 15 in Table 1 for country scores of relational mobility). May relational mobility moderate the strength of preference for family over personal well-being? Further studies on family well-being with more countries are needed to test this hypothesis.

Our study also supports previous research that shows that the extent to which well-being is valued significantly and strongly varies across countries: from not far above the neutral point (i.e., Japan) to almost hitting the ceiling of the valuation scale (i.e., Colombia), with the difference between these two countries reaching over one standard deviation. Our additional results reported in the online supplementary material, which show significant positive associations between valuing and experiencing each type of well-being, suggest that low levels of actual well-being in Confucian countries may be the effect of moderate valuations of well-being, and high levels of actual well-being in Latin American countries may be the effect of their elevated valuations of well-being. Said differently, valuing well-being may be an important antecedent for experiencing well-being.

In three of four analyzed countries, we also found that valuation of relationship-oriented well-being (i.e., interdependent happiness) is as high as valuation of achievement-oriented well-being (i.e., life satisfaction). This pattern is somewhat unexpected as interdependent happiness was conceptualized as particularly relevant to Eastern cultures. Most notably, Japanese participants seemed to value personal satisfaction and interdependent happiness similarly, as did Canadian and Colombian participants. This is a signal that further studies on interdependent happiness are necessary to unpack these findings, and expand our understanding of this construct as a complement to the large body of research on life satisfaction.

Well-being seems to be the 'obvious ultimate dependent variable' in psychological studies. Our research documents, however, that things might be more complex. If we want to treat well-being as the ultimate aim of human and societal flourishing, we first may need to answer questions about which type of well-being and whose flourishing we should assess. The answer to this question will probably vary depending on the cultural context. Thus, here, we propose that in order to deliver a more cross-culturally generalizable picture on human flourishing, researchers may need to study various kinds of well-being concurrently (e.g., Krys et al. 2019). We also posit that further studies on indigenous conceptions of well-being are necessary.

Furthermore, studies on tensions between personal and family well-being seem to be necessary too. Personal wellbeing and the well-being of one's family do not have to be in conflict (North et al. 2008). However, most people sometimes face the dilemma of whether to focus on their own wellbeing or on the well-being of their family. The item from the vertical collectivism subscale 'It is my duty to take care of my family, even when I have to sacrifice what I want' (Singelis 
et al. 1995) illustrates the personal-family well-being tension. In contrast, these two types of well-being may be complementary: personal well-being may contribute to family well-being, and family well-being may be a fundament of personal wellbeing. The conditions under which personal and family wellbeing are complementary or incompatible can be a subject of further empirical studies. ${ }^{5}$

\section{Limitations}

These advances notwithstanding, it is important to acknowledge our study's shortcomings. For instance, the studied group - students - may differ from the general population in how they value different types of well-being. It is plausible that young adults may value family well-being less than other age cohorts. Once students graduate, for instance, they often set up their own families and would probably place more value on family well-being at this stage of life compared to when they were students. These assumptions need empirical verification though. Next, to keep the design simple and consistent across countries and individuals, the scales we employed were not counterbalanced-future studies may need to repeat our findings with the scales rotated. Another potential limitation is that two of the samples we studied turned out to be gender imbalanced-further studies may need to be based on more gender-balanced samples. Ideally, it would be great to incorporate items on family well-being into large cross-country studies carried out on representative samples (e.g., WVS). The current study focused on two concepts of well-being: life satisfaction and interdependent happiness. We selected them because we wanted to analyze well-being in a cross-cultural context and these two concepts mirror cultural differences in the conceptualization of well-being. But other family-related concepts of well-being need to be studied too. In particular, relationship flourishing (Fowers et al. 2016) seems to be a concept requiring additional attention in cross-cultural studies on family well-being.

We are aware that culture is a far more complex construct than how we use it in the current paper. The crosscountry comparability of our data can be disputed due to the lack of full scalar equivalence or possible response

\footnotetext{
$\overline{5}$ We propose that different time perspectives may help analyse this puzzle. Whereas in short-term decisions personal and family well-being can sometimes compete, in the long run these two seem to be highly complementary (family well-being fosters personal well-being, and personal well-being fosters family well-being). Another perspective that may help is the theoretical distance between the analysed kinds of well-being: the further the specific kind of well-being is from the another, the larger tensions between them one may expect to find (e.g., from among the four types of well-being analysed in the current research, the largest tensions, if existent at all, should be between personal life satisfaction and family interdependent happiness).
}

biases (see $\mathrm{He}$ et al. 2017). But even if our cross-country findings suffer from insufficient comparability, we still reliably document that in each of the four analyzed countries family well-being is valued more than personal well-being. Had each culture been analyzed separately, this main conclusion would be the same. Lastly, future studies should try to recruit samples from other countries; pan-cultural generalizations drawn from data collected in four countries need to be done with caution.

\section{Concluding Remarks}

The majority of previous studies implicitly assume that all people prefer to be well, but fewer studies (Hornsey et al. 2018; Koh 2014) explicitly acknowledge that people may value wellbeing to various extents. Even if different well-beings contribute to each other, people (in particular, from various cultural backgrounds) do differently value various types of well-being. Thus, future studies on well-being valuation seem to be necessary. How does well-being valuation change over the lifespan? Why do people in certain cultures value well-being highly vs. moderately? What is the evolutionary function of well-being valuation? How does the gap between actual and valued levels of well-being influence human behavior? These are only a few questions that remain to be answered.

By recognizing in its resolution from 2011 that well-being is a fundamental human goal, the UN accelerated discussion on the directions of societal development. Here, we would like to make an argument in this discussion: in pursuing well-being, policy makers may need to not only analyze personal well-being, but the well-being of a family as a whole as well (see also Krys et al. 2019). Our study documents that, across four countries, it is family well-being that is valued over personal well-being. We suggest that family as a subject of wellbeing measures needs to be taken into account in subsequent studies. Similarly, cultural differences in the valuation of different types of well-being also need special attention. After all it seems obvious that we all want to be well, but how much we desire well-being, which type of well-being, and for whomfor ourselves or for our families - demands further empirical studies.

Funding Details This work was supported by the Polish National Science Centre under grant 2016/23/D/HS6/02946; and the Japan Society for the Promotion of Science under grants P17806 and 17F17806.

Data Availability Data and syntax supporting the results can be downloaded from the Open Science Framework at https://osf.io/ 9fbwp/?view_only=7f2cfb0fb491414c81d4fc7117ce936f

\section{Compliance with Ethical Standards}

Conflict of Interest On behalf of all authors, the corresponding author states that there is no conflict of interest. 
Open Access This article is distributed under the terms of the Creative Commons Attribution 4.0 International License (http:// creativecommons.org/licenses/by/4.0/), which permits unrestricted use, distribution, and reproduction in any medium, provided you give appropriate credit to the original author(s) and the source, provide a link to the Creative Commons license, and indicate if changes were made.

\section{References}

Adler, A., \& Seligman, M. E. P. (2016). Using wellbeing for public policy: Theory, measurement, and recommendations. International Journal of Wellbeing, 6, 1-35.

Andersz, N., Czarnota-Bojarska, J., \& Wojtkowska, K. (2018). Strategies for maintaining work-life balance and their consequences for quality of life and job satisfaction. Social Psychological Bulletin, 13, 1-13.

Baker, W. E. (2014). United America: The surprising truth about American values, American identity and the 10 beliefs that a large majority of Americans hold dear. Ann Arbor: Read the Spirit Books.

Bond, M. H. (2002). Reclaiming the individual from Hofstede's ecological analysis-a 20-year odyssey: Comment on Oyserman et al. (2002). Psychological Bulletin, 128, 73-77.

Bowman, R. A. (2001). Quality of life assessment for young children with developmental disabilities and their families: Development of a quality of life questionnaire (Doctoral dissertation). West Virginia University, Morgantown, WV.

Brislin, R. (1970). Back-translation for cross-cultural research. Journal of Cross-Cultural Psychology, 1, 185-216.

Brown, I., Brown, R. I., Baum, N. T., Isaacs, B. J., Myerscough, T., Neikrug, S., ... Wang, M. (2006) Family quality of life survey general version. Surrey Place Centre, Toronto, Canada.

Chien, N. C., \& Mistry, R. S. (2013). Geographic variations in cost of living: Associations with family and child well-being. Child Development, 84, 209-225.

Cousins, S. D. (1989). Culture and self-perception in Japan and the United States. Journal of Personality and Social Psychology, 56, 124-131.

DeFrain, J., \& Asay, S. M. (2007). Family strengths and challenges in the USA. Marriage \& Family Review, 41, 281-307.

Delle Fave, A., Brdar, I., Wissing, M. P., Araujo, U., Castro Solano, A., Freire, T., et al. (2016). Lay definitions of happiness across nations: The primacy of inner harmony and relational connectedness. Frontiers in Psychology, 7, 1-23.

Diener, E., Emmons, R. A., Larsen, R. J., \& Griffin, S. (1985). The satisfaction with life scale. Journal of Personality Assessment, 49, 71-75.

Diener, E., Diener, M., \& Diener, C. (1995). Factors predicting the subjective well-being of nations. Journal of Personality and Social Psychology, 69, 851-864.

Diener, E., Napa-Scollon, C. K., Oishi, S., Dzokoto, V., \& Suh, E. M. (2000). Positivity and the construction of life satisfaction judgments: Global happiness is not the sum of its parts. Journal of Happiness Studies, 1, 159-176.

Diener, E., Oishi, S., \& Lucas, R., (2015) National accounts of subjective well-being. American Psychologist, 70, 234-242.

Fowers, B. J., Laurenceau, J.-P., Penfield, R. D., Cohen, L. M., Lang, S. F., Owenz, M. B., \& Pasipanodya, E. (2016). Enhancing relationship quality measurement: The development of the relationship flourishing scale. Journal of Family Psychology, 30, 997-1007.

He, J., Van de Vijver, F., Fetvadjiev, V., Dominguez Espinosa, A., Adams, B., Alonso-Arbiol, I., ... Mõttus, R. (2017) On enhancing the crosscultural comparability of likert-scale personality and value measures: A comparison of common procedures. European Journal of Personality, 31, 642-657.

Helliwell, J., Layard, R., \& Sachs, J. (2016). World happiness report 2016. Retrieved October 4, 2018 from http://worldhappiness. report .

Henrich, J., Heine, S. J., \& Norenzayan, A. (2010). The weirdest people in the world? Behavioral and Brain Sciences, 33(1-23), 61-83.

Hitokoto, H., \& Uchida, Y. (2015). Interdependent happiness: Theoretical importance and measurement validity. Journal of Happiness Studies, 16, 211-239.

Hofstede, G. (2001). Culture's consequences: Comparing values, behaviors, institutions, and organizations across nations, second edition. Thousand Oaks: Sage Publications, Inc..

Hornsey, M., Bain, P., Harris, E., Lebedeva, N., Kashima, E., Guan, Y., Gonzalez, R., Chen, S., \& Blumen, S. (2018). How much is enough in a perfect world? Cultural variation in ideal levels of happiness, pleasure, freedom, health, self-esteem, longevity, and intelligence. Psychological Science, 29, 1393-1404.

Hu, X., Summers, J. A., Turnbull, A., \& Zuna, N. (2011). The quantitative measurement of family quality of life: A review of available instruments. Journal of Intellectual Disability Research, 55, 1098-1114.

Johnson, H., Zabriskie, R., \& Hill, B. (2006). The contribution of couple leisure involvement, leisure time, and leisure satisfaction to marital satisfaction. Marriage and Family Review, 40, 69-91.

Kamp Dush, C. M., Taylor, M. G., \& Kroeger, R. A. (2008). Marital happiness and psychological well-being across the life course. Family Relations, 57, 211-226.

Koh, S. (2014). The (un)desirability of happiness: Pathogen threats predict differences in the value of happiness (Doctoral dissertation). Retrieved September 7, 2016 from http://ink.library.smu.edu.sg/ .

Krys, K., Uchida, Y., Oishi, S., \& Diener, E. (2018). Open society fosters satisfaction: Explanation to why individualism associates with country level measures of satisfaction. The Journal of Positive Psychology. Advance online publication. https://doi.org/10.1080/ 17439760.2018.1557243.

Krys, K., Zelenski, J. M., Capaldi, C. A., Park, J., van Tilburg, W., van Osch, Y., ... Uchida, Y. (2019). Putting the "we" into well-being: Using collectivism-themed measures of well-being attenuates wellbeing's association with individualism. Asian Journal of Social Psychology. Advance online publication. https://doi.org/10.1111/ ajsp.12364.

Lakens, D. (2013). Calculating and reporting effect sizes to facilitate cumulative science: A practical primer for t-tests and ANOVAs. Frontiers in Psychology, 4, 1-12.

Lee, H., Stewart, S., Lun, V., Bond, M., Yu, X., \& Lam, T. (2012). Validating the concord index as a measure of family relationships in China. Journal of Family Psychology, 26, 906-915.

Lu, L., \& Gilmour, R. (2004). Culture and conceptions of happiness: Individual oriented and social oriented SWB. Journal of Happiness Studies, 5, 269-291.

Markus, H. R., \& Kitayama, S. (1991). Culture and the self: Implications for cognition, emotion, and motivation. Psychological Review, 98, 224-253.

McKeown, K., \& Sweeney, J. (2001). Family well-being and family policy: A review of research on benefits and costs. Retrieved August 7, 2018 from http://kieranmckeown.ie/wp-content/uploads/2013/03/ 15.-Family-well-being-and-family-policy.pdf .

Newland, L. A. (2015). Family well-being, parenting, and child wellbeing: Pathways to healthy adjustment. Clinical Psychologist, 19, $3-14$.

North, R. J., Holahan, C. J., Moos, R. H., \& Cronkite, R. C. (2008). Family support, family income, and happiness: A 10 -year perspective. Journal of Family Psychology, 22, 475-483.

Olson, D. H., \& Wilson, M. A. (1982). Family satisfaction scale. In D. H. Olson, H. I. McCubbin, H. L. Barnes, A. S. Larsen, M. J. Muxen, \& 
M. A. Wilson (Eds.), Family inventories (pp. 25-31). St. Paul: Family Social Science, University of Minnesota.

Olson, D., Portner, J., \& Lavee, Y. (1985). Family adaptability and cohesion evaluation scale (FACES III). St Paul: University of Minnesota.

Park, J., Hoffman, L., Marquis, J., Turnbull, A. P., Poston, D., Hamman, H., et al. (2003). Toward assessing family outcomes of service delivery: Validation of a family quality of life survey. Journal of Intellectual Disability Research, 47, 367-384.

Ryff, C. D., \& Keyes, C. L. M. (1995). The structure of psychological well-being revisited. Journal of Personality and Social Psychology, 69, 719-727.

Sampson, E. E. (1981). Cognitive psychology as ideology. American Psychologist, 36, 730-743.

Singelis, T. M. (1994). The measurement of independent and interdependent self-construals. Personality and Social Psychology Bulletin, 20, 580-591.

Singelis, T. M., Triandis, H. C., Bhawuk, D. P. S., \& Gelfand, M. J. (1995). Horizontal and vertical dimensions of individualism and collectivism: A theoretical and measurement refinement. CrossCultural Research, 29, 240-275.

Smilkstein, G. (1978). The family APGAR: A proposal for a family function test and its use by physicians. Journal of Family Practice, $6,1231-1239$.
Thomson, R., Yuki, M., Talhelm, T., Schug, J., Kito, M., Ayanian, A. H., et al. (2018). Relational mobility predicts social behaviors in 39 countries and is tied to historical farming and threat. Proceedings of the National Academy of Sciences, 115, 7521-7526.

Uchida, Y., \& Kitayama, S. (2009). Happiness and unhappiness in east and west: Themes and variations. Emotion, 9, 441-456.

Uchida, Y., \& Oishi, S. (2016). The happiness of individuals and the collective. Japanese Psychological Research, 58, 125-141.

Ura, K., Alkire, S., Zangmo, T., \& Wangdi, K. (2012). A short guide to gross national happiness index. Bhutan: The Centre for Bhutan Studies.

World Values Survey. (2016). Online data analysis. Retrieved from http:// www.worldvaluessurvey.com.

Zabriskie, R. B., \& McCormick, B. P. (2003). Parent and child perspectives of family leisure involvement and satisfaction with family life. Journal of Leisure Research, 35, 163-189.

Zabriskie, R. B., \& Ward, P. J. (2013). Satisfaction with family scale. Marriage \& Family Review, 49, 446-463.

Publisher's note Springer Nature remains neutral with regard to jurisdictional claims in published maps and institutional affiliations. 